\title{
قياس أثر الفاقد على الموارد الاقتصادية الزراعية والأمن الغذائي لأهم محاصيل الحبوب في مصر
}

\author{
سحر عبد المنعم السيد قمره، أمل أحمد فؤاد جميلة، غادة صالح حسن

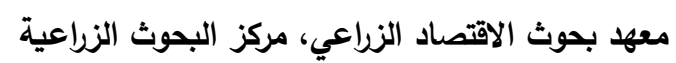

Received: Mar. 8 , 2020

Accepted: May 4, 2020

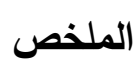

استهذف البحث التعرف على البعد الاقتصادي للفاقد ومدى ارتباطه بالفقد في الموارد الاقتصادية الزراعية والأمن الغذائي

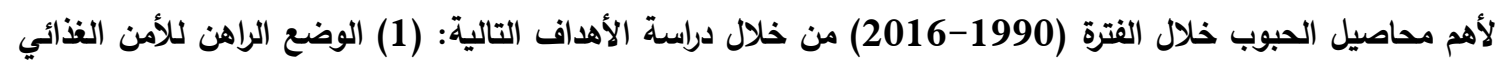

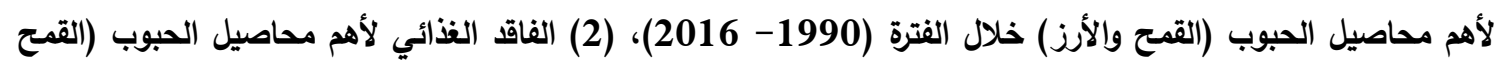
والأرز) ومدى إرتباطه بالفقد في الموارد الاقتصادية الزراعية وذلك من خلال دراسة (أ) تطور كمية الفاقد ونسبته إلى الإنتاج والإستهلاك لمحاصيل الدراسة خلال الفترة (2010-2016) ، (ب) تقدير قيمة الخسارة الاقتصادية نتيجة الفقد في الموارد

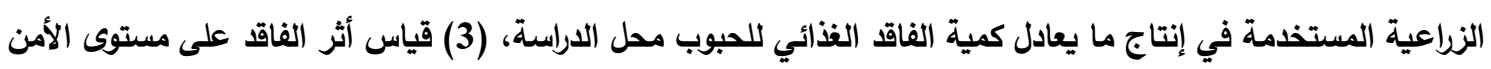
الغذائي لأهم محاصيل الحبوب (القدح والأرز) خلال فترة الدراسة.

إعتمد البحث في تحقيق أهدافه على المعادلات الاقتصادية المستخدمة في تقدير الفقد في الموارد الاقتصادية الزراعية

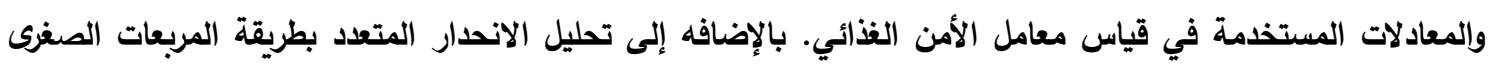
العادية (OLS). (المعادلات

وقد توصل البحث إلى مجموعة من النتائج أهمها: (1) بدراسة الوضع الراهن للأمن الغذائي لأهم محاصيل الحبوب، تبين أن المخزون الإستراتيجي لكل من القدح والأرز بلغ حوالي 10,11، 10.

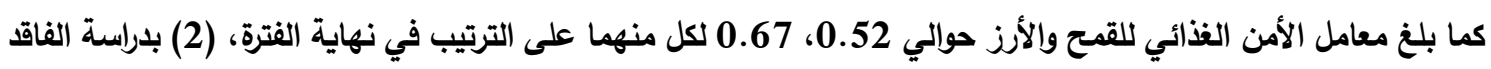

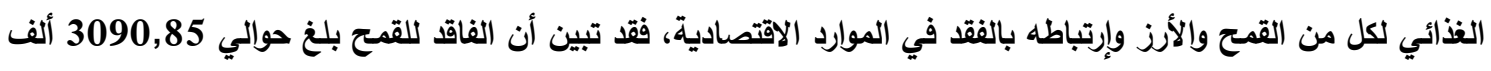

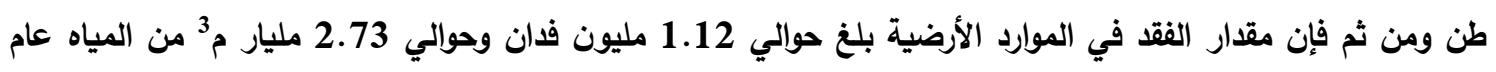

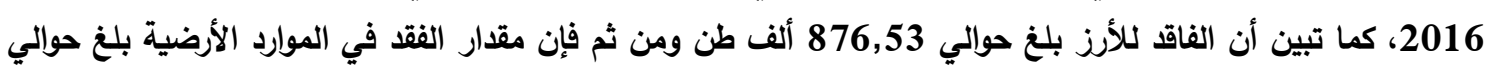

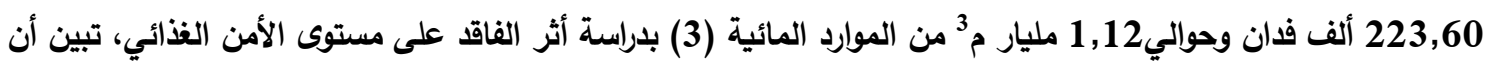

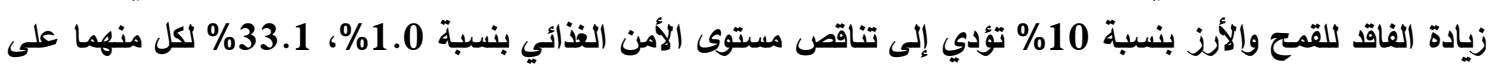

وتوصي هذه الاراسة بضرورة الد من الفاقد الغذائي وآثاره السلبية على الموارد الاقتصادية الزراعية والأمن الغذائي،

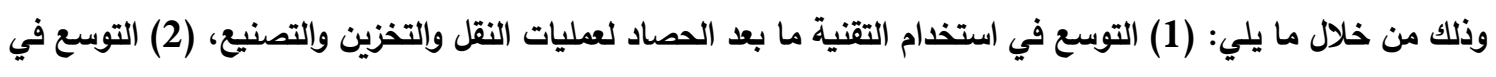

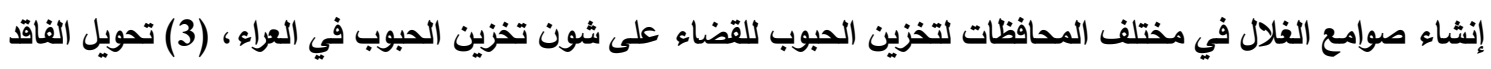
الغذائي إلى أعلاف للماشية في حالة علدم ملائمته للاستهلاك الآدمي. كلمات دالة: الفاقد، الخسائر الاقتصادية للموارد الاقتصادية الزراعية، الأمن الغذائي، القمح والأزز. 


$$
\text { والززاعة، 2016). }
$$

وأشارت بعض الاراسات إلى أن ما يقرب من ثلث

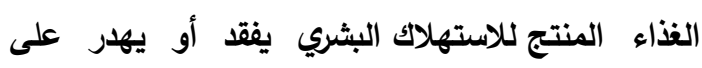
المستوى العالمي، حيث يبلغ حوالي 3,1 مليار طن سنوياً. وهذا يعني أن كميات هائلة من الموارد المستخدمة في ملئي

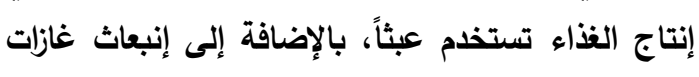
الاحتباس الحراري الناجمة عن إنتاج الأغذية التي يتم التباء

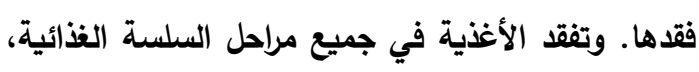

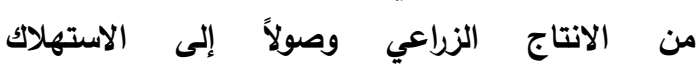
الأسري. ويهار الغذاء في البلدان المتوسطة والمرتفعة

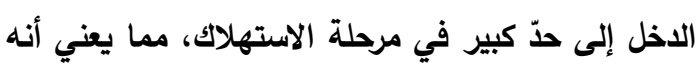

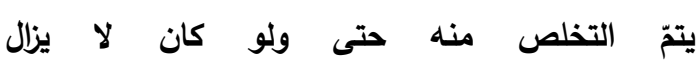

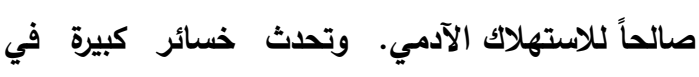

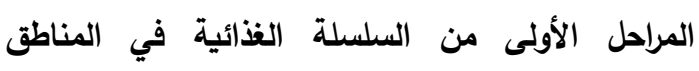

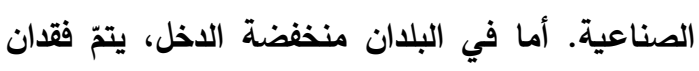

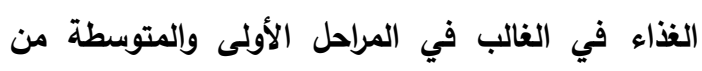

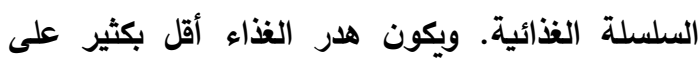
مستوى المستهلك. وترتبط أسباب الفاقد والهلدر الغذائي في البلدان منذفضة الدخل بشكل أساسي بالقيود المالية

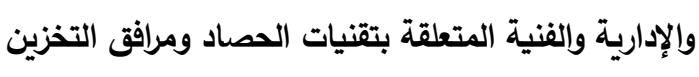
والتبريد، وفي ظروف جوية صعبة وفي البنية التحتية ونظم التعبئة والتسويق. ونظراً لأن العديد من المزارعين من وفي النية التئية

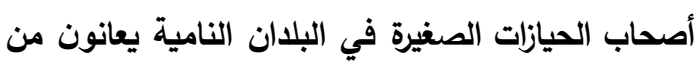

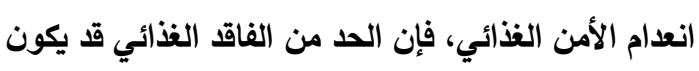
له تأثير فوري وكبير على سبل معيثتهر. وقدرت قيمة الخسائر الاقتصادية والاجتماعية نتيجة الفقد في الموارد الأرضية والعمالة الزراعية والموارد المائية والأسمدة الكيماوية بحوالي 120,23 مليون جنيه في محافظة الإسكندرية (قمره، 2008). وباتت السياوية بهاسية التسويقية لأهم الخضروات والفاكهة في حاجة إلى تقليل المخاطرة الطبيعية (الفاقد التسويقي) وذلك من خلال تطبيق تقنية ما بعد الحصاد خلال المراحل التسويقية ولية
إهتمت مصر بالمواصفات القياسية للسلع والخدمات

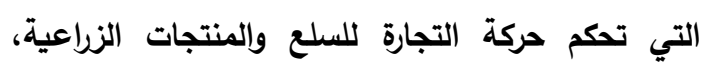
بالإضافة إلى اهتمامها بإنثاء وتطوير الأجهزة الحكومية

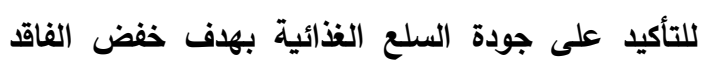
وتعزيز القدرة التنافسية للمنتجات الزراعية أمام مثيلتها

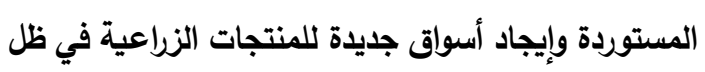
المتغيرات والتطورات الاقتصادية العالمية بعد تحرير التجارة

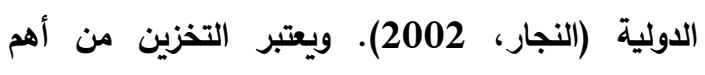
الوظائف والخدمات التسويقية، نظراً لموسمية الإنتاج الزراعي واستمرارية الطلب على السلع الزراعية طوال

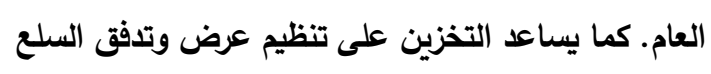

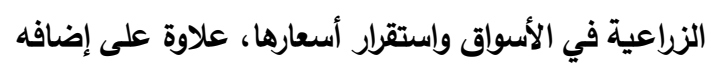

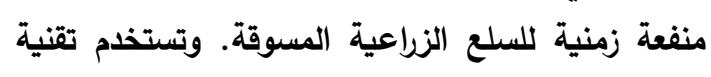

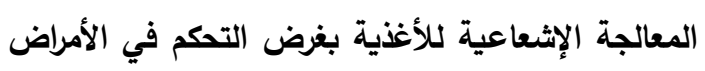
التي تصيب الأغذية وإطالة فترة التخزين، كما تستخدم كبديل للمطهرات والكيماويات التي تترك بقايا ضارة على التى الأغذية. وفي عام 1997 صدر القرار رقم 3220 المتعلق بالمواصفات القياسية المصرية للمعالجة بالإثعاع، حيث الأبثان

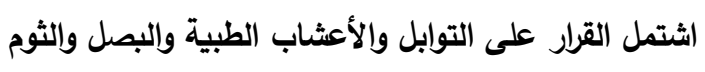
المجفف، ثم صرح بعد ذلك بمعالجة البطاطس والبطاطا (النجار، 2002).

وتتم معظم العمليات التسويقية للسلع الزراعية بالطرق

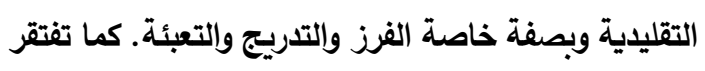
الأسواق في مصر إلى وسائل النقل والتخزين المبرد

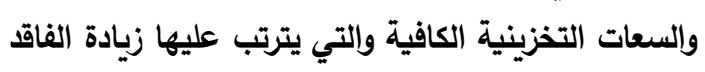
التسويقي، والذي يعتبر جزء مستقطع من إجمالي الربح

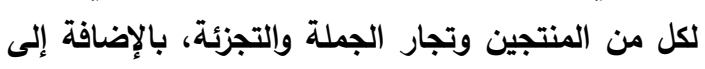
الخسائر الاقتصادية والاجتماعية نتيجة الفقد في الموارد

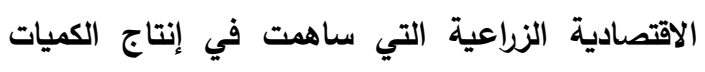
المفقودة من السلع الغذائية. ويقدر الفاقد من الأغذية والهلار الغذائي في منطقة الثرق الأدنى وشمال أفريقيا بما يصل إلى حوالي 250 كيلو جرام للفرد الواحد سنوياً 
الفاقد من أهم السلع الاستراتيجية. وتتمثل تلك

$$
\text { المعادلات فيما يلي (قمره، 2008): }
$$

1- مقدار الفقد في الموارد الأرضية (المساحة المزروعة) (200ل)

= (كمية الفاقد : متوسط إنتاجية الفدان).

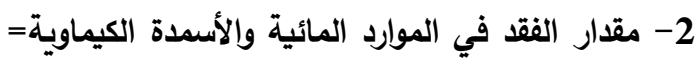

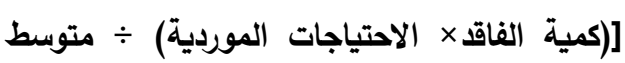

$$
\text { إنتاجية الفدان]. }
$$

(ب) المعادلات الاقتصادية المستخدمة في قياس معامل الأمن الغذائي. وتتمثل تلك المعادلات فيما يلي (غانم،

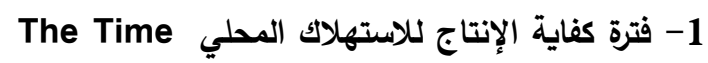
Interval of productions

$$
\text { المحلي ٪ الاستهلاك المحلي اليومي. }
$$

The Time فترة تغطية الواردات للاستهلاك المحلي اليوهي Interval of Sufficiency

$$
\text { الاستهلاك المحلي اليومي. }
$$

3- مقدار الفائض والعجز في الإستهلاك = [(مجموع طول

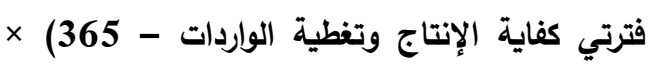

الاستهلاك المحلي اليومي) -كمية الصادرات].

4-معامل الأمن الغذائي= المخزون الاستراتيجي (محصلة الائل

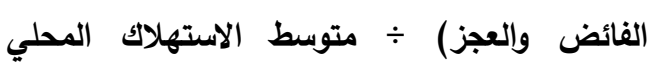

السنوي. كما يمكن تقديره من خلال محصلة نسبة

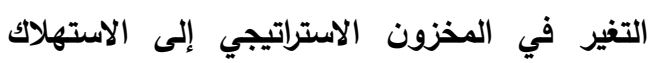

$$
\text { المحلي السنوي. }
$$

وتتراوح قيمة معامل الأمن الغذائي بين الصفر والواحد

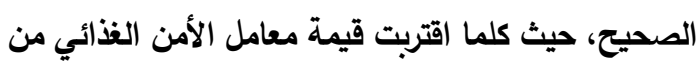

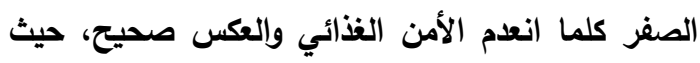

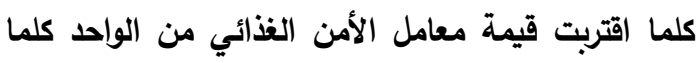
ازداد مستوى الأمن الغذائي للسلعة في الدولة (غانم وقمره،

(ج) تحليل الانحدار المتعدد لقياس أثر الفاقد على الأمن

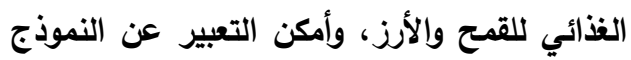

المقدر بالمعادلة التالية:

$Y_{t}=b_{0}+b_{1} X_{1 t}+b_{2} X_{2 t}++b_{3} X_{3 t}+e_{t}$
المتمثلة في الفرز والتدريج، التعبئة، النقل، التخزين

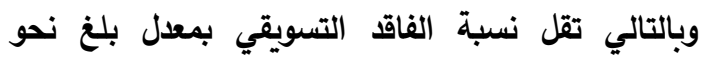
20.27\% ومما لا شك فيه أن الفاقد الإنتاجي والتسويقي والاستهلاكي يدل على وجود كميات من المواد الغذائية لا يتم الاستفادة منها في كل من المناطق الحضرية والريفية ولاكية

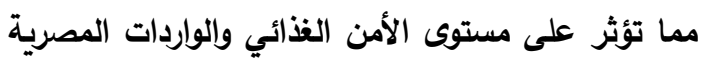
لأهم السلع الغذائية الاستراتيجية.

الأهداف البحثية: إستهاف البحث التعرف على البعد الاقتصادي للفاقد الغذائي ومدى ارتباطه بالفقد في الموارد الاقتصادية الزراعية والأمن الغذائي لأهم محاصيل الحبوب (القدح والأرز) خلال الفترة (1990-2016)، وذلك من خلال

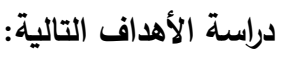
1- الوضع الراهن للأمن الغذائي لأهم محاصيل الحبوب (القـح والأرز) خلال الفترة (1990- 2016).

2- الفاقد الغذائي لأهم محاصيل الحبوب (القدح والأرز) ومدى إرتباطه بالفقد في الموارد الاقتصادية الزراعية وذلك من خلال دراسة (أ) تطور كمية الفاقد ونسبته إلى الإنتاج والإستهلاك لمحاصيل الدارة داسلة خلال الفترة (2010-2016) ، (ب) تقدير قيمة الخسارة الاقتصادية نتيجة الفقد في الموارد الزياعية المستخدمة في إنتاج ما يعادل كمية الفاقد الغذائي

$$
\text { للحبوب محل الاراسة. }
$$

3- قياس أثر الفاقد على مستوى الأمن الغذائي لأهم محاصيل الحبوب (القمح والأرز) خلال فترة الدراسة.

$$
\text { الأسلوب البحثي: - إعثد }
$$

إعتمد البحث في تحقيق أهدافه على التحليل

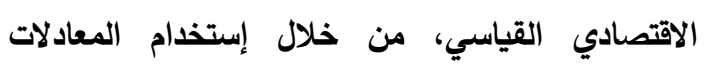
والنماذج التالية: (أ) المعادلات الاقتصادية المستخدمة في تقدير الفقد في الموارد الاقتصادية الزراعية (الأرض والعمالة والمياه والأسمدة الكيماوية) المستخدمة في إنتاج ما يعادل الزراعلة الأرضال والماه 
عام 2006، ثم تناقصت إلى حوالي 175.8 يوم عام 2016. كما تراجعت فترة تغطية الواردات للاستهلاك المحلي للقدح من حوالي 227.3 يوم عام 1990، إلى لـ حوالي 106.0 يوم عام 2009، ثم ازدادت إلى حوالي 164.2 يوم عام 2016. ويعتبر زيادة فترة كفاية الإنتاج وتراجع فترة تغطية الواردات للاستهلاك المحلي للقدح مؤشر جيد في صالح الاقتصاد المصري، حيث يمكن من خلال الاستمرار في تحقيق هذا الهذف حماية الاقتصاد

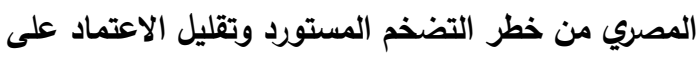
الخارج ومن ثم تخفيف العجز في الميزان التجاري الزراعي Balance of المصري وبالتالي ميزان المدفوعات Payments، بالإضافة إلى حماية الأمن الغذائي لمصر ميران من التقلبات الاقتصادية والسياسية والمناخية للدول المحتكرة لإنتاج وتصدير القـح في العالم. تم جمع فائض surplus من القمح عن الاستهلاك المحلي خلال السنوات 1990-1992، 1994، 1998-1996، 2000، 2002، 2008-2005، 2015-2010، حيث قر إجمالي الفائض بقرابة 16.05 مليون طن خلال فترة الاراسة، في حين حدث عجز Deficit في القمح المخصص للاستهلاك المحلي خلال السنوات الأخرى، حيث قدر إجمالي العجز بنحو 4.94 مليون طن خلال فترة الدراسة. ووفقاً لمفهوم المخزون الاستراتيجي باعتباره محصلة كل من الفائض صلون والعجز خلال فترة الدراسة، فقد تبين أنه بلغ حوالي 10.11 مليون طن، وفي ضوء الاستهلاك المحلي للقمح البالغ حوالي 19.41 مليون طن عام 2016، يقدر معامل الأمن الغذائي للقمح بحوالي 0.52 في نهاية الفترة.
حيث أن: تمثل مستوى الأمن العذائي المقدر للسلعة، (X) تمثل فترة كفاية الإنتاج للاستهلاك المحلي، (X)

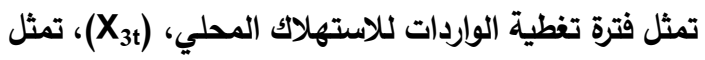
مقدار الفاقد الغذائي. وتم تقدير النموذج بطريقة المربعات

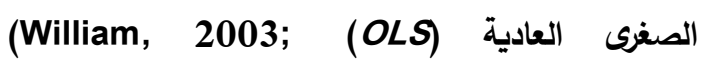
.Gujarati, 1979)

$$
\text { مصادر البيانات البحثية: }
$$

اعتمدت هذه الدراسة في تحقيق أهدافها على البيانات

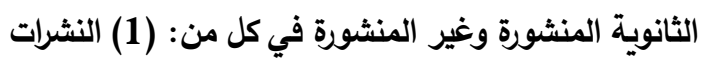

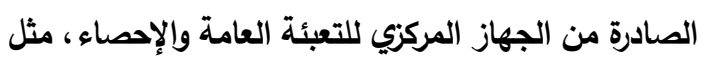
الكتاب الإحصائي السنوي، النشرة السنوية لإحصاء الري

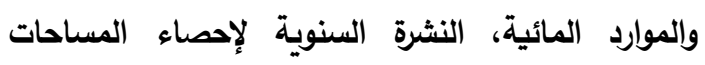
المحصولية والإنتاج النباتي، النشرة السنوية لحركة الإنتاج والتجارة الخارجية والمتاح للاستهلاك من السلع الزراعية، نشرة مصر في أرقام، (2) الموقع الإكتروني لمنظمة

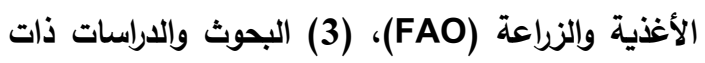

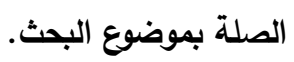

$$
\text { النتائج البحثية }
$$

أولاً: الوضع الراهن للأمن الغذائي لأهم محاصيل الحبوب (القمح والأرز) خلال الفترة (1990-

(2016 الوضع الراهن للأمن الغذائي للقمح: بدراسة الوضع الراهن للأمن الغذائي للقدح من خلال تقدير المخزون الاستراتيجي وحساب مقدار الفائض والعجز في القمح المخصص للاستهلاك المحلي خلال الفترة (12016-1990). تبين من البيانات الواردة بجدول زيادة فترة كفاية الإنتاج للاستهلاك المحلي للقدح من لينال

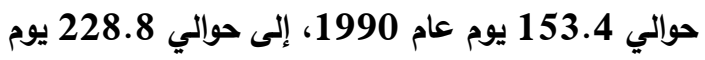
جدول (1): تطور فترتي كفاية الإنتاج وتغطية الواردات للاستهلاك المحلي ومقدار التغير في المخزون الاستراتيجي ومعامل

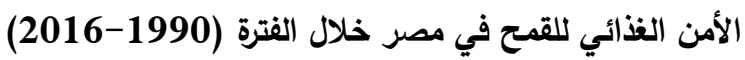

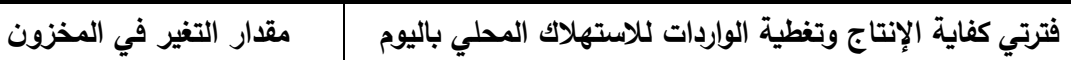


Measuring the impact of waste on agricultural economic resources and food .....

\begin{tabular}{|c|c|c|c|c|c|c|}
\hline \multicolumn{2}{|c|}{ الاستراتيجي بالألف طن } & \multirow{2}{*}{ الفترتين } & \multirow{2}{*}{ فترة تغطية الواردات } & \multirow{2}{*}{ فترة كفاية الإنتاج } & \multirow{2}{*}{ الاسلهي اليوميلاكي } & \multirow[t]{2}{*}{ السنة } \\
\hline 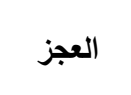 & الفائض & & & & & \\
\hline- & 437.1 & 380.7 & 227.3 & 153.4 & 27.83 & 1990 \\
\hline- & 431.6 & 380.5 & 219.7 & 160.8 & 27.87 & 1991 \\
\hline- & 32.0 & 366.1 & 201.3 & 164.8 & 28.02 & 1992 \\
\hline 407.4 & - & 350.4 & 176.9 & 173.5 & 27.85 & 1993 \\
\hline- & 488.5 & 381.2 & 234.1 & 147.1 & 30.17 & 1994 \\
\hline 92.2 & - & 362.0 & 176.2 & 185.8 & 30.79 & 1995 \\
\hline- & 250.3 & 372.9 & 191.9 & 181.0 & 31.68 & 1996 \\
\hline- & 960.7 & 394.5 & 214.7 & 179.9 & 32.52 & 1997 \\
\hline- & 159.0 & 370.0 & 177.3 & 192.8 & 31.61 & 1998 \\
\hline 372.1 & - & 352.7 & 143.1 & 209.6 & 30.28 & 1999 \\
\hline- & 310.3 & 375.1 & 161.0 & 214.1 & 30.65 & 2000 \\
\hline 1270.6 & - & 326.3 & 135.6 & 190.7 & 32.81 & 2001 \\
\hline- & 459.8 & 379.4 & 173.6 & 205.8 & 32.20 & 2002 \\
\hline 595.7 & - & 346.1 & 129.0 & 217.2 & 31.52 & 2003 \\
\hline 410.3 & - & 352.5 & 133.5 & 219.0 & 32.78 & 2004 \\
\hline- & 817.7 & 387.8 & 161.1 & 226.6 & 35.93 & 2005 \\
\hline- & 911.1 & 390.2 & 161.4 & 228.8 & 36.16 & 2006 \\
\hline- & 52.7 & 366.6 & 163.2 & 203.4 & 36.28 & 2007 \\
\hline- & 2145.4 & 420.3 & 214.8 & 205.5 & 38.82 & 2008 \\
\hline 1428.8 & - & 327.9 & 106.0 & 221.9 & 38.40 & 2009 \\
\hline- & 49.5 & 366.0 & 218.2 & 147.8 & 48.55 & 2010 \\
\hline- & 1014.6 & 386.6 & 208.5 & 178.1 & 47.00 & 2011 \\
\hline- & 1895.0 & 402.2 & 229.6 & 172.6 & 50.96 & 2012 \\
\hline- & 400.0 & 372.6 & 191.1 & 181.5 & 52.33 & 2013 \\
\hline- & 3413.8 & 438.2 & 239.2 & 199.0 & 46.64 & 2014 \\
\hline- & 1820.1 & 401.3 & 210.9 & 190.5 & 50.44 & 2015 \\
\hline 1366.5 & - & 340.0 & 164.2 & 175.8 & 53.17 & 2016 \\
\hline 4943,6 & 16049,2 & \multicolumn{5}{|c|}{ الإجمالي } \\
\hline \multicolumn{2}{|c|}{10105.6} & \multicolumn{5}{|c|}{ المخزون الاستراتيجي } \\
\hline \multicolumn{2}{|c|}{0.52} & \multicolumn{5}{|c|}{ معامل الأمن الغذائي } \\
\hline
\end{tabular}

المصدر: جمعت وحسبت من:

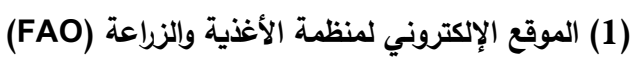
(2) الجهاز المركزي للتعبئة العامة والإحصاء، الكتاب الإحصائي السنوي، 2018.

والعجز في الأرز المخصص للاستهلاك المحلي خلال الفترة

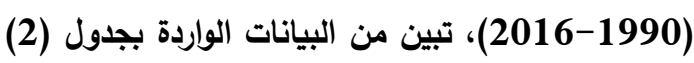

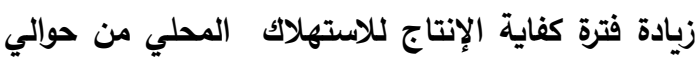

الوضع الراهن للأمن الغذائي للأرز : براسة الوضع الراهن للأمن الغذائي للأرز في مصر من خلال تقدير المخزون الاستراتيجي ومقدار الفائض 
بدراسة تطور الفاقد للقمح ونسبته إلى الإنتاج والاستهلاك خلال الفترة (2010- 2016)، تبين من البيانات الواردة بجدول (3) أن كمية الفاقد تراوح بين حد أدنى بلغ حوالي 1800,76 ألف طن عام 2010 وحد أقصى بلغ حوالي 3230,07 ألف طن عام 2014، بمتوسط سنوي قر بحوالي 2737,88 ألف طن. وتراوحت نسبة الفاقد إلى الإنتاج المحلي بين حد أدنى بلغ نحو 24,72\% عام 2010 وحد أعلى بلغ نحو \%34.99 عام 2014، بمتوسط سنوي بلغ نحو 30.90 خلال فترة الاراسة. كما تراوحت نسبة الفاقت إلى الاستهلاك المحلي بين حد أدنى بلغ نحو 10.16 عام 2010 وحد أعلى بلغ نحو 18.98\% عام 2014، بمتوسط سنوي بلغ نحو 15.04\% خلال فترة الدراسة. وبلراسة تظور الفاقد للأرز ونسبته إلى الإنتاج والاستهلاك خلال الفترة (2010- 2016)، تبين من البيانات الواردة بجدول (4) أن كمية الفاقد تراوح بين حد أدنى بلغ حوالي 956,56 ألف طن عام 2014 وحد أقصى بلغ حوالي 1267,85 ألف طن عام 2010، بمتوسط سنوي قرر بحوالي 1071,88 ألف طن. وتراوحت نسبة الفاقد إلى الإنتاج المحلي بين حد أدنى بلغ نحو 16,19\% عام 2014 وحد أعلى بلغ نحو 23.79\% عام 2010، بمتوسط سنوي بلغ نحو 19.19\% خلال فترة الاراسة. كما تراوحت نسبة الفاقد إلى الاستهلاك المحلي بين حد أدنى بلغ نحو 17,03\% عام 2014 وحد أعلى بلغ نحو 24.14\% عام 2011، بمتوسط سنوي بلغ نحو 20.48\% خلال فترة الاراسة.

374.1 يوم عام 1990، إلى حوالي 479.3 يوم عام 2008، ثم تناقصت إلى حوالي 363.6 يوم عام 2016. أما بالنسبة للواردات فقد ساهمت بنسبة ضئيلة، حيث تراوحت فترة تغطية الواردات للاستهلاك المحلي بين حد أدنى بلغ حوالي 0.02 يوم عام 1992، وحد أعلى بلغ حوالي 19.4 يوم عام 2012.

تم جمع فائض من الأرز عن الاستهلاك المحلي خلال معظم السنوات للفترة (1990-2016)، حيث بلغ إجمالي الفائض 6.81 مليون طن وتم توجيه هذا الفائض لتنمية المخزون الاستراتيجي للأزز. كما حدث عجز في الأرز المخصص للاستهلاك المحلي خلال السنوات 1991، 1998 حيث بلغ إجمالي العجز 3.55 مليون طن، وتم تغطية العجز من خلال السحب من المخزون الاستراتيجي للأزز. تفوق مقدار الفائض الموجه لتنمية المخزون الاستراتيجي للأرز على مقدار العجز، حيث بلغت نسبة مقدار الفائض إلى العجز 191.7\% في نهاية الفترة (1990-2016). ووفقاً لمفهوم المخزون الاستراتيجي باعتباره محصلة كل من الفائض والعجز خلال فترة الاراسة، قر المخزون الاستراتيجي للأزز بنحو 3.26 مليون طن. وفي ضوء الاستهلاك المحلي للأرز البالغ حوالي 4.84 مليون طن عام 2016، يقدر معامل الأمن الغذائي بنحو 0.67 في نهاية فترة الدراسة. ثانياً: الفاق الغذائي لأهم محاصيل الحبوب (القمح والأرز) ومدى ارتباطه بالفقد في الموارد الاقتصادية الزراعية تطور كمية الفاقد ونسبته إلى الإنتاج والاستهلاك لمحاصيل الدراسة خلال

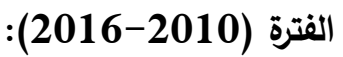
جدول (2): تطور فترتي كفاية الإنتاج وتغطية الواردات للاستهلاك المحلي ومقدار التغير في المخزون الاستراتيجي ومعامل الأمن الغذائي للأرز في مصر خلال الفترة (1990-

\begin{tabular}{|c|c|c|c|c|}
\hline \multirow{2}{*}{ مقدار التغير في المخزون } & \multicolumn{2}{|c|}{ فترتي كفاية الإنتاج وتغطية الواردات للاستهلاك المحلي باليوم } & \multirow{2}{*}{ المحلي اليومي } & \multirow[b]{2}{*}{ السنة } \\
\hline & فترة تغطية الواردات & فترة كفاية الإنتاج & & \\
\hline
\end{tabular}


Measuring the impact of waste on agricultural economic resources and food .....

\begin{tabular}{|c|c|c|c|c|c|c|}
\hline العزز & الفائض & مجموع الفترتين & & & بالألف طن & \\
\hline - & 3.60 & 374.39 & 0.29 & 374.1 & 8.47 & 1990 \\
\hline 30.1 & - & 378.22 & 0.42 & 377.8 & 9.13 & 1991 \\
\hline- & 165.0 & 401.22 & 0.02 & 401.2 & 9.75 & 1992 \\
\hline- & 181.0 & 395.93 & 0.13 & 395.8 & 10.51 & 1993 \\
\hline- & 193.3 & 403.79 & 0.09 & 403.7 & 11.35 & 1994 \\
\hline- & 183.8 & 392.89 & 0.39 & 392.5 & 12.20 & 1995 \\
\hline- & 19.2 & 392.83 & 0.03 & 392.8 & 12.46 & 1996 \\
\hline- & 702.3 & 437.16 & 0.06 & 437.1 & 12.54 & 1997 \\
\hline 428.7 & - & 365.06 & 0.06 & 365.0 & 12.26 & 1998 \\
\hline- & 793.5 & 450.01 & 0.51 & 449.5 & 12.94 & 1999 \\
\hline- & 763.9 & 452.19 & 0.09 & 452.1 & 13.27 & 2000 \\
\hline 94.1 & - & 408.05 & 7.55 & 400.5 & 13.05 & 2001 \\
\hline- & 198.1 & 409.39 & 0.09 & 409.3 & 14.92 & 2002 \\
\hline- & 233.4 & 420.20 & 0.13 & 420.7 & 14.68 & 2003 \\
\hline- & 287.5 & 443.24 & 0.21 & 443.3 & 14.33 & 2004 \\
\hline- & 189.7 & 463.35 & 0.34 & 463.1 & 13.23 & 2005 \\
\hline- & 652.1 & 479.19 & 7.39 & 471.8 & 14.32 & 2006 \\
\hline- & 363.6 & 472.17 & 7.97 & 464.2 & 14.82 & 2007 \\
\hline- & 1414.3 & 479.62 & 0.59 & 479.3 & 15.13 & 2008 \\
\hline 603.4 & - & 356.9 & 0.83 & 356.7 & 15.48 & 2009 \\
\hline 1890.9 & - & 273.92 & 0.88 & 273.4 & 15.84 & 2010 \\
\hline- & 3.0 & 368.7 & 7.8 & 360.9 & 12.00 & 2011 \\
\hline- & 466.38 & 396.7 & 19.4 & $\mathbf{3 7 7 . 3}$ & 15.04 & 2012 \\
\hline 286.94 & - & 400.5 & 1.4 & 399.1 & 14.81 & 2013 \\
\hline 70.63 & - & 373.9 & 2.0 & 371.9 & 15.39 & 2014 \\
\hline 102.90 & - & 382.3 & 2.0 & 379.3 & 14.41 & 2015 \\
\hline 47.20 & - & 365.9 & 2.3 & 363.6 & 13.25 & 2016 \\
\hline 3554.87 & 6813.68 & \multicolumn{5}{|c|}{ الإجمالي } \\
\hline \multicolumn{2}{|c|}{3258.81} & \multicolumn{5}{|c|}{ المخزون الاستراتيجي } \\
\hline \multicolumn{2}{|c|}{0.67} & \multicolumn{5}{|c|}{ معامل الأمن الغذائي } \\
\hline
\end{tabular}

المصدر: جمعت وحسبت من:

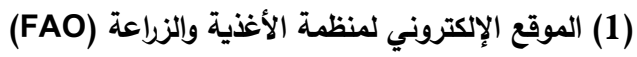
(2) الجهاز المركزي للتعبئة العامة والإحصاء، الكتاب الإحصائي السنوي، الإعيه جدول (3): تطور كمية الفاقد بالألف طن للقمح ونسبته إلىى الإنتاج والاستهلاك خلال الفترة (2010- 2016)

\begin{tabular}{|c|c|c|c|c|c|}
\hline \multicolumn{2}{|c|}{ الأهمية النسبية للفاق % } & \multirow[t]{2}{*}{ الاستهلاك } & \multirow[t]{2}{*}{ الإنتاج } & \multirow[t]{2}{*}{ الفاقد } & \multirow[t]{2}{*}{ 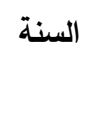 } \\
\hline الاستهلاك & الإنتاج & & & & \\
\hline 10.16 & 24,72 & 17720,75 & 7284,35 & 1800,76 & 2010 \\
\hline
\end{tabular}


Sahar Kamara, et al.,

\begin{tabular}{|c|c|c|c|c|c|}
\hline 13.26 & 26,87 & 17155,00 & 8469,01 & 2275,66 & 2011 \\
\hline 14.82 & 31.06 & 18600,40 & 8875,13 & 2756,55 & 2012 \\
\hline 15.07 & 30.35 & 19100,45 & 9480,90 & 2878,06 & 2013 \\
\hline 18.98 & 34.99 & 17023,60 & 9231,00 & 3230,07 & 2014 \\
\hline 17.02 & 31.86 & 18410,60 & 9833,25 & 3132,63 & 2015 \\
\hline 15.93 & 29.96 & 19407,05 & 10314,90 & 3090,85 & 2016 \\
\hline 15.04 & 30.90 & 18102.55 & 9069,79 & 2737,88 & المتوسط \\
\hline 2.80 & 3.35 & 928.98 & 992.87 & 522.03 & الانحراف المعياري \\
\hline 18.64 & 11.09 & 5.10 & 10.95 & 19.06 & معامل الاختلاف \% \\
\hline
\end{tabular}

المصدر: جمعت وحسبت من: الجهاز المركزي للتعبئة العامة والإحصاء، النشرة السنوية لحركة الإنتاج والتجارة الخارجية والمتاح للاستهلاك من السلع الزراعية، أعداد متفرقة، الفترة 2010- 2016.

جدول (4): تطور كمية الفاقد بالألف طن للأرز ونسبته إلى الإنتاج والاستهلاك خلال الفترة (2010- 2016)

\begin{tabular}{|c|c|c|c|c|c|}
\hline \multicolumn{2}{|c|}{ الأهمية النسبية للفاقد \% } & \multirow[t]{2}{*}{ الاستهلاك } & \multirow[t]{2}{*}{ الإلتاج } & \multirow[t]{2}{*}{ 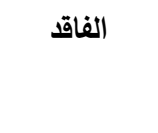 } & \multirow[t]{2}{*}{ 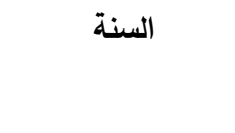 } \\
\hline الاستهلاك & الإنتاج & & & & \\
\hline 21.93 & 23,79 & 5781,60 & 5328,28 & 1267,85 & 2010 \\
\hline 24.14 & 18,67 & 4380,00 & 5664,18 & 1057,35 & 2011 \\
\hline 21.43 & 20.30 & 5489,60 & 5794,45 & 1176,54 & 2012 \\
\hline 18.27 & 17.00 & 5405,65 & 5809,00 & 987,55 & 2013 \\
\hline 17.03 & 16.19 & 5617,35 & 5900,00 & 956,56 & 2014 \\
\hline 22.45 & 21.60 & 5259,65 & 5465,50 & 1180,76 & 2015 \\
\hline 18.12 & 16.75 & 4836,25 & 5234,30 & 876,53 & 2016 \\
\hline 20.48 & 19.19 & 5252.87 & 5600,82 & 1071,88 & المتوسط \\
\hline 2.67 & 2.83 & 487.73 & 260.57 & 141.42 & الانحراف المعياري \\
\hline 13.01 & 14.79 & 9.29 & 4.65 & 13.19 & معامل الاختلاف \% \\
\hline
\end{tabular}

المصدر: جمت وحسبت من: الجهاز المركزي للتعبئة العامة والإحصاء، النشرة السنوية لحركة الإنتاج والتجارة الخارجية والمتاح للاستهلاك من السلع الزراعية، أعداد متفرقة، الفترة 2010- 2016.

بحساب مقار الفقد في الموارد الاقتصادية الزراعية في ضوء الاحتياجات الموردية وكمية الفاقد الغذائي، تبين من البيانات الواردة بجدول (5) أنه بالنسبة للقدح فإن مقدار الفاقد بلغ حوالي 3090,85 ألف طن ومتوسط لجن
تقدير قيمة الخسارة الاقتصادية نتيجة الفقد في الموارد الزراعية المستخدمة في إنتاج ما يعادل كمية الفاقد الغذائي للحبوب محل الاراسة: 
النموذج في الصورة الخطية ونصف اللوغاريتمية. وتبين أفضلية النموذج الخطي في تمثيل البيانات المستخدمة في

التقدير وأمكن التعبير عنه بالمعادلة التالية: $\hat{Y}_{1}=-1.002+0.003 X_{1}+0.002 X_{2}-0.0000002 X_{3}$

$(-712.69)^{* *}(554.79)^{* *}(828.04)^{* *}(-2.39)^{*}$

$R^{2}=0.99 \quad F=252448.6$ D. $W=1.45$

LM test $=0.77$ Arch test $=0.50$

تبين من النموذج المقدر أن زيادة فترتي كفاية الإنتاج وتغطية الواردات للاستهلاك المحلي بنسبة 10\% تؤدي إلى زيادة مستوى الأمن الغذائي للقمح بنسبة 240.4\% 155.1\% لكل منهما على التوالي"، في حين زيادة حجم الفاقد للقمح بنسبة 10\% تؤدي إلى تناقص مستوى الأمن الغذائي للقدح بنسبة 1.0\%. وتقدر قيمة معامل التحديد (R2) 0.99 وهوالي يعني أن المتغيرات المستقلة التي يتضمنها النموذج تفسر 99\% من التغيرات التي حثت في مستوى الأمن الغذائي للقـح خلال فترة الدراسة، بينما بقية التغيرات وتقدر بحوالي 1\% ترجع إلى عوامل أخرى لا يتضمنها النموذج المقدر. ومن خلال الاختبارات الإحصائية للنموذج المقترح، يتضح أنه خالي من مشكلة الارتباط الذاتي للبواقي، حيث الاحتبه

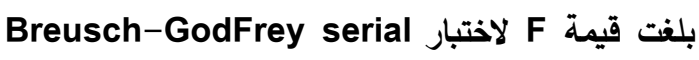
correlation LM Test حوالي 0.77 وهى غير معنوية إحصائياً عند المستوى الاحتمالي 1\%، مما يال على خلو النموذج المقدر من مشكلة الارتباط الذاتي للبواقي. كما يتمتع النموذج المقدر بكفاءة جيدة في تمثيل البيانات

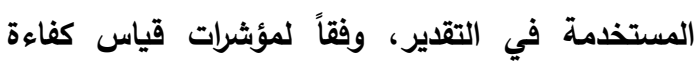
النموذج وأهمها معامل عدم التساوي لثيل (U- Theil) والذي اقتربت قيمته من الصفر · جدول (6).
إنتاجية الفان بلغ حوالي 2.77 طن وحيث أن الاحتياجات المائية حوالي 2450 م²/ فدان، فإن الطن المنتج من القمح يتطلب كمية من المياه تبلغ حوالي 884.48 م² طن. ومن ثم فإن مقدار الفقد في الموارد الأرضية قدر بحوالي 1.12 مليون فدان ومقدار الفقد في الموارد المائية قدر بحوالي 2.73 مليار م³ عام 2016.

كما تبين أن مقدار الفاقد للأرز بلغ حوالي 876,53 ألف طن ومتوسط إنتاجية الفدان بلغ حوالي 3,92 طن الني

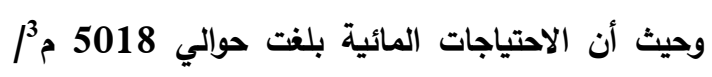
فدان، فإن الطن المنتج من الأرز يتطلب كمية من المياه

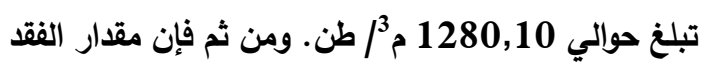
في الموارد الأرضية قدر بحوالي 223,60 ألف فدان ومقدار الفقد في الموارد المائية قدر بحوالي 1,12 مليار

$$
\text { م } 3 \text { عام } 2016 .
$$

ثالثاً: قياس أثر الفاقد على مستوى الأمن الغذائي لأهم محاصيل الحبوب (القـح والأرز) خلال الفترة (1990-2016) قياس أثر الفاقد على مستوى الأمن الغذائي للقمح: يتحدد مستوى الأمن الغذائي للقمح معبراً عنه بنسبة مقدار الفاقد والعجز إلى الاستهلاك المحلي بمجموعة من المتفيرات وأهمها فترة كفاية الإنتاج للاستهلاك المحلي وفترة تغطية الواردات للاستهلاك المحلي (X) بالإضافة إلى مقدار الفاقد بالألف طن (X) أثر الفاقد على مستوى الأمن الغذائي للقدح من خلال تقدير نموذج الانحدار المتعدد بطريقة المربعات الصغرى الصني العادية (OLS) خلال الفترة (1990- 2016). ونظراً لوجود بعض القيم السالبة في نسبة مقدار الفائض والعجز إلى الاستهلاك المحلي (المتغير التابع)، فقد تم تقدير لمعليم

$$
\begin{aligned}
& \text { * يتم تقدير المرونة في النموذج الخطي من خلال القانون } \\
& \text { التالي: }
\end{aligned}
$$


3.7\% لكل منهما على التوالي"، في حين زيادة حجم

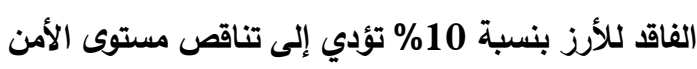

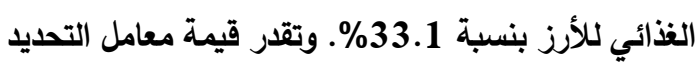

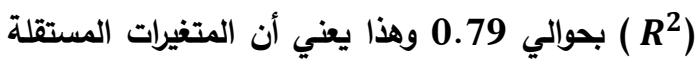
التي يتضمنها النموذج تفسر 79\% من ولت التغيرات التي

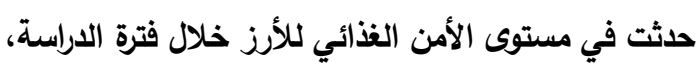

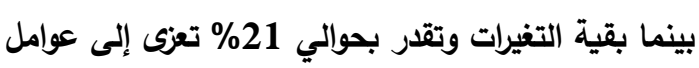
أخرى لا يتضمنها النموذج المقدر. ومن خلال الاختبارات الإحصائية للنموذج المقترح،

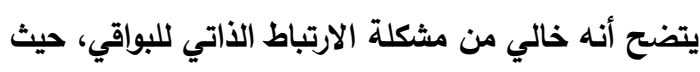

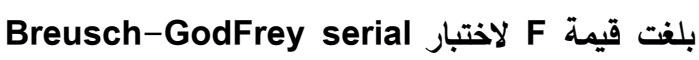
correlation LM Test حوالي 1.02 وهى غير معنوية إحصائياً عند المستوى الاحتمالي 1\%، مما يدل على خلو

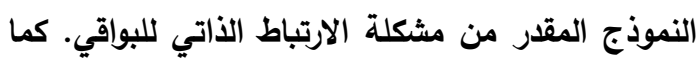
يتمتع النموذج المقدر بكفاءة جيدة في تمثيل البيانات

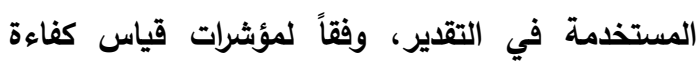
النموذج وأهمها معامل عدم التساوي لثيل (U- Theil) والذي اقتربت قيمته من الصفر .جدول (7).
قياس أثر الفاقد على مستوى الأمن الغذائي للأرز: بقياس أثر الفاقد على مستوى الأمن الغذائي للأرز

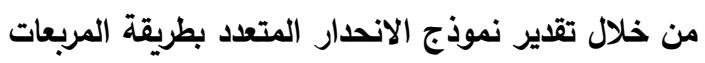

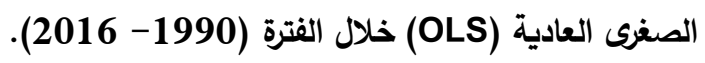

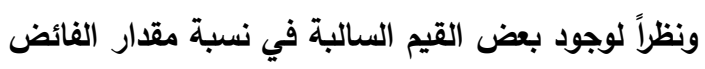
والعجز إلى الاستهلاك المحلي (المتغير التابع)، فقد تم تقدير النموذج في الصورة الخطية والنصف لوغاريتمية. وتبين أفضلية النموذج الخطي في تمثيل البيانات المستخدمة في التقدير وأمكن التعبير عنه فيلة فالمعادلة التالية:

$$
\begin{aligned}
\hat{Y}_{2}= & -84.65+0.24 X_{1}+0.42 X_{2}-0.03 X_{3} \\
& (-8.84)^{* *}(8.30)^{* *}(1.98)^{*}(-1.96)^{*} \\
& R^{2}=0.79 \quad F=28.15 \quad D . W=1.93
\end{aligned}
$$

LM test $=1.02$ Arch test $=0.94$
وتبين من النموذج المقدر أن زيادة فترتي كفاية الإنتاج

وتغطية الواردات للاستهلاك المحلي بنسبة 10\% تؤدي

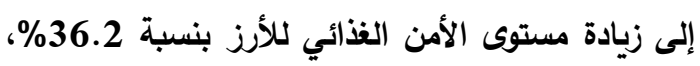

جدول (5): مقدار الفقد في الموارد الأرضية والمائية المرتبط بالفاقد الغذائي للمحاصيل محل الدراسة عام 2016 مقدار الفقد في الموارد 
Measuring the impact of waste on agricultural economic resources and food .....

\begin{tabular}{|c|c|c|c|c|c|c|}
\hline المائية بالمليون & فالأرضية بالألف & متوسط نصيب & الاحتياجية مُ الفات & طنوسط الإنتاجية & كمية الفاقد & الحبوب \\
\hline 2733.78 & 1115.83 & 884.48 & 2450 & 2.77 & 3090,85 & القــح \\
\hline 1122,05 & 223,60 & 1280,10 & 5018 & 3,92 & 876,53 & الأرز \\
\hline
\end{tabular}

المصدر: جمعت وحسبت من:

1- الجهاز المركزي للتعبئة العامة والإحصاء، النشرة السنوية لإحصاء الري والموارد المائية عام 2016، إصدار ديسمباء العباء ، 2017.

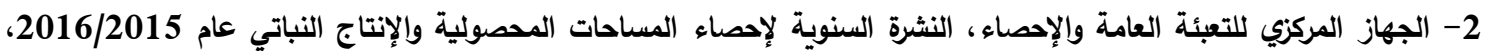

إصدار يناير، 2018.

جدول (6): مؤشرات قياس كفاءة النموذج المقدر للقمح خلال الفترة (1990- 2016)

\begin{tabular}{|c|c|}
\hline 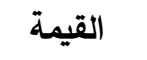 & المؤشر \\
\hline 0.0004 & R.M.S.E. الجذر التربيعي لمتوسط مربعات الخطأ العشوائي \\
\hline 0.0003 & M.A.E. متوسط الخطأ المطلق \\
\hline 1.63 & M.A.P.E. متوسط النسبة المئوية للخطأ المطلق \\
\hline 0.002 & معامل عدم التساوي لثيل Theil (U) \\
\hline
\end{tabular}

المصدر: جمعت وحسبت من النموذج المقدر بالبحث.

جدول (7): مؤشرات قياس كفاءة النموذج المقدر للأرز خلال الفترة 1990- 2016

\begin{tabular}{|c|c|}
\hline 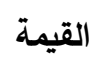 & المؤشر \\
\hline 4.86 & R.M.S.E. الجذر التربيعي لمتوسط مربعات الخطأ العشوائي \\
\hline 3.99 & M.A.E. متوسط الخطأ المطلق \\
\hline 49.1 & M.A.P.E. متوسط النسبة المئوية للخطأ المطلق \\
\hline 0.23 & (U) Theil معامل عدم التساوي لثيل \\
\hline
\end{tabular}

المصدر: جمعت وحسبت من النموذج المقدر بالبحث.

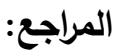


8- غانم، عادل محمد خليفة وسحر عبد المنعم قمره (2010). دراسة العوامل الاقتصادية المحددة لمعامل

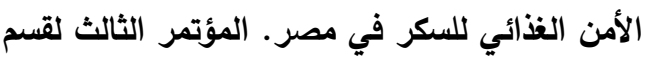
الإقتصاد وإدارة الأعمال الزراعية (إستراتيجية التنمية

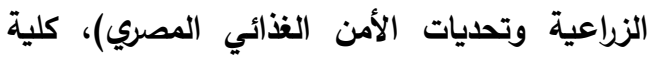
الزراعة، جامعة الإسكندرية، (28- 29) يوليو، مجلة

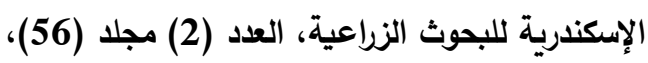
عدد خاص، أغسطس، 2011، ص: 1- 10. 9- قمره، سحر عبد المنعم (2008). أثر التقنية ما بعد ألد

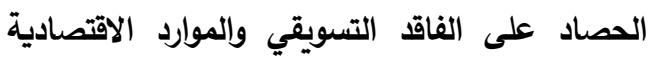
الزراعية لأهم الخضروات والفاكهة المنتجة بمحافظة الفاقل الإسكندرية، مجلة جامعة المنصورة للعلوم الزراعية، كلية الزراعة، جامعة المنصورة، مجلد (33)، العدد

$$
\text { (5)، مايو. }
$$

10- منظمة الأغذية والزراعة، الموقع الإلكتروني

$$
\text { (FAOSTAT) }
$$

11- Makridakis, S., S. Wheelwrights and V.E. McGee (1993). Forecasting Methods and Application. $2^{\text {nd }}$ ed New York: Johns Wiley and Sons.

12- William, H. Greene (2003). Econometric Analysis, Fifth edition, New York University.
1- الجهاز المركزي للتعبئة العامة والإحصاء، الكتاب

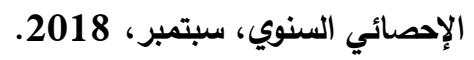

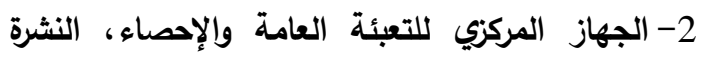
السنوية لإحصاء الري والموارد المائية عام 2016،

$$
\text { إصدار ديسمبر } 2017 .
$$

3- الجهاز المركزي للتعبئة العامة والإحصاء، النشرة

السنوية لإحصاء المساحات المحصولية والإنتاج

النباتي عام 2016/2015، إصدار يناير، 2018.

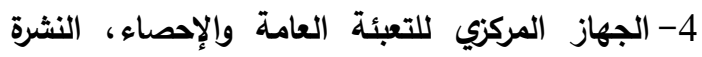

السنوية لحركة الإنتاج والتجارة الخارجية والمتاح

للاستهلاك من السلع الزراعية، أعداد متفرقة، الفترة

$$
\text { 2016-2010 }
$$

5- الجهاز المركزي للتعبئة العامة والإحصاء، نشرة مصر

$$
\text { في أرقام } 2018 .
$$

6- النجار، أحلام (2002). تنسيق المواصفات القياسية

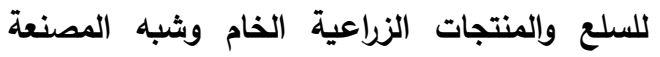

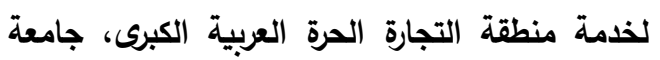
الدول العربية، المنظمة العربية للتنمية الزراعية،

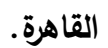
7- غانم، عادل محمد خليفة (1997). قضية الأمن

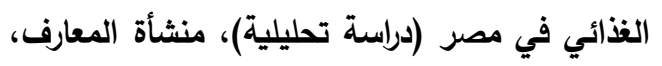
الإسكندرية. 
Measuring the impact of waste on agricultural economic resources and food .....

\title{
MEASURING THE IMPACT OF WASTE ON AGRICULTURAL ECONOMIC RESOURCES AND FOOD SECURITY FOR THE MOST IMPORTANT CEREAL CROPS IN EGYPT
}

\author{
Sahar Kamara, Amal F. Jamila and Ghada S. Hassan \\ Agricultural Economics Research Institute, Agricultural Research Center
}

\begin{abstract}
The research aimed to identify the economic dimension of the loss and its relationship to the loss in agricultural economic resources and food security of the most important cereal crops in Egypt during the period (1990-2016) by studying the following objetives: (1) the current status of food security of the most important cereal crops (wheat and rice) during the period (1990) - 2016), (2) The nutritional loss of the most important cereals crops (wheat and rice) and its extent related to the loss in agricultural economic resources, through a study (a) the evolution of the amount of loss and its ratio to production and consumption of the study cereals crops during the period (2010-2016), (b) an estimate of the value of the economic loss as a result of the loss in the agricultural resources used in the production The equivalent of the amount of waste Alimentarius for cereals, (3) to measure the impact of losses on the level of food security for the most important cereal crops (wheat and rice) during the study period.

This study relied on achieving its objectives, on the economic equations used in estimating the loss in the agricultural economic resources and the equations used in measuring the food security coefficient. This study also relied on the multiple regression analysis by using ordinary least squares method (OLS).

The research reached a set of results, the most important of which are: (1) By studying the current state of food security of the most important cereal crops, it was found that the strategic stocks of both wheat and rice amounted to about 10.11, 3.26 million tons during the period (1990-2016), and reached The coefficient of food security for wheat and rice is $0.52,0.67$ each, respectively at the end of the period, (2) By studying the nutritional loss of both wheat and rice and its correlation with the loss in economic resources, it has been shown that the loss of wheat amounted to 3090.85 tons, and then the amount of loss in land resources amounted to about 1.12 million feddans and about 2.73 billion $\mathrm{m}^{3}$ of water in 2016, as it was found that the loss of rice It amounted to 876.53 thousand tons, so the amount of the loss in the land resources amounted to about 223.60 thousand feddans and about 1.12 billion $\mathrm{m}^{3}$ of water resources, (3) by studying the impact of losses on the level of food security, it was found that increasing the losses of wheat and rice by $10 \%$ leads to a decrease in the level of food security by $1.0 \%, 33.1 \%$ each, respectively.

Finally, this study recommends the need to reduce food losses and its negative effects on agricultural economic resources and food security, through the following: (1) the expansion of the use of post-harvest technology for transport, storage and manufacturing operations, (2) the expansion of the establishment of grain silos in various governorates to store grains, for eliminate cereals storing in the open, (3) converting food losses into fodder for livestock in case they are not suitable for human consumption.
\end{abstract}

Key words: Waste, economic losses of agricultural economic resources, food security, wheat and rice.

$$
\begin{aligned}
& \text { السادة المحكمين } \\
& \text { أ.د/ جابر بسيونى كلية الزراعة - جامعة سابا باشا }
\end{aligned}
$$

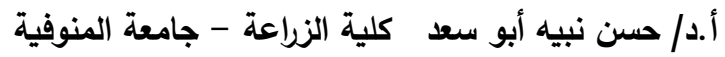


